\title{
Factores de riesgo para hipoglucemia neonatal transitoria en pacientes a término, estudio de casos y controles en un hospital de Bogotá
}

\author{
Risk factors for transient neonatal hypoglycemia in term individuals, case \\ and control study in a hospital of Bogotá
}

\section{Cristo Colmenares J, ${ }^{1}$ Burbano Camacho E, ${ }^{2}$ Ortiz CS, ${ }^{3}$ Gómez Hoyos D. ${ }^{4}$}

\author{
${ }^{1} \mathrm{MD}$, pediatra neonatólogo, jefe de servicio, Hospital Universitario \\ Clínica San Rafael. Bogotá, Colombia. \\ ${ }^{2} \mathrm{MD}$, residente de pediatría, Universidad Militar Nueva granada. \\ Bogotá, Colombia. \\ ${ }^{3} M D$, residente de pediatría, Universidad Militar Nueva Granada. \\ Bogotá, Colombia. \\ ${ }^{4} \mathrm{MD}$, pediatra neonatóloga, Hospital Universitario Clínica San \\ Rafael. Bogotá, Colombia.
}

Autor de correspondencia: Eduardo Burbano Camacho

Correo electrónico: edburbanoc@gmail.com

Fecha de recepción: 20/04/2020

Fecha de aceptación: 13/02/2021

\section{Resumen}

Introducción: la hipoglucemia neonatal es frecuente y puede presentarse de forma transitoria o persistente. Existen diferentes puntos de corte de glucemia para poder definirla y usualmente son menores de $47 \mathrm{mg} / \mathrm{dL}$. Se han evidenciado factores de riesgo tanto maternos como neonatales para desarrollar esta enfermedad, que aumentan el riesgo de padecerla entre un $14,7 \%$ y 83,2 \% en comparación a un 5\% respecto a los pacientes que no los poseen. Así mismo se ha demostrado que el retraso en el tratamiento de la hipoglucemia genera alteraciones en el neurodesarrollo.

Objetivo: determinar los factores de riesgo asociados con hipoglucemia neonatal transitoria de recién nacidos a término sin factores de riesgo determinados por la guía de la Academia Americana de Pediatría, en el Hospital Universitario Clínica San Rafael en el período comprendido de enero de 2015 a enero de 2019.

Metodología: estudio observacional analítico, retrospectivo de tipo casos y controles, desde enero de 2015 a enero de 2019. Se realiza el cálculo de muestra y se obtienen 35 casos y 35 controles mediante el software estadístico Real Statistics 6.9 de enero de 2020.

Resultados: La mayor proporción de pacientes en el estudio fue de sexo femenino (61,43\%), 20 casos y 23 controles. El promedio de glucosa de ingreso de los casos fue de 40,8 mg/ dL. Dentro de los factores de riesgo se encontró el sobrepeso materno (OR de 1,2), cesáreas (OR 2,5), hipertensión inducida por el embarazo (OR 4,38). Se encontró una asociación significativa con Apgar al minuto de 5 (3 pacientes [8,57 \%]); primer aporte energético recibido del recién nacido (25 [71,4\%] recibieron lactancia materna), taquipnea transitoria del recién nacido (6 [17,14\%]) y policitemia (10 [28,57 \%]) como variables en el recién nacido y el tiempo de apego como variable materna (7 [20\%]) en los casos que no la recibieron.

Conclusiones: en la población a término considerada sana que desarrolla hipoglucemia neonatal transitoria se encontró asociación con un Apgar al minuto de 5, el primer aporte energético recibido, la taquipnea transitoria del recién nacido, la policitemia y el apego como factores que influyen en su aparición. Se hace un llamado para hacer una mayor vigilancia de este grupo de recién nacidos que, aunque presentan menor incidencia de hipoglucemia, sí necesitan del acompañamiento y seguimiento.

Palabras clave: hipoglucemia; factores de riesgo; recién nacido.

\begin{abstract}
Introduction: Hypoglycemia in newborns is a frequent pathology, and it may present as transient or persistent. Many different cut-off points are used to define blood glucose, usually set at less than $47 \mathrm{mg} / \mathrm{dL}$. There is evidence for both maternal and neonatal risk factors that increase the risk of developing the disease by $14.7 \%$ to $83.2 \%$, as compared to $5 \%$ in patients
\end{abstract}


without those risk factors. Likewise, it has been demonstrated that delay in the treatment of hypoglycemia is associated with neurodevelopmental alterations.

Objective: To determine the risk factors associated with transient neonatal hypoglycemia in term infants with no risk factors as defined by the American American Academy of Pediatrics guidelines, at Hospital Universitario Clínica San Rafael during the period between January, 2015 and January, 2019.

Methodology: Observational, analytical, retrospective case-control study conducted between January, 2015 and January, 2019. Sample calculation was performed and 35 cases and 35 controls were obtained using the Real Statistics software 6.9 of January, 2020.

Results: The highest proportion of patients in the study was female (61.43\%), 20 cases and 23 controls. Average glucose level on admission of the cases was $40.8 \mathrm{mg} / \mathrm{dL}$. Risk factors included maternal overweight with an odds ratio (OR) of 1.2; caesarean section, performed in 15 cases (42.8\%), with an OR of 2.5; and pregnancy-induced hypertension, found in 4 cases and 1 control, with an OR of 4.38. There was evidence of a significant association with an Apgar score of 5 in the first minute in 3 cases (8.57\%); the first energy intake by the newborn, with 25 (71.4\%) of the cases receiving breastfeeding; transient tachypnea of the newborn (TTN) in $6(17.14 \%)$ cases; and polycythemia in 10 (28.57\%) cases, as neonatal variables, and skin-to-skin contact as a maternal variable in 7 cases (20\%) that did not receive it.

Conclusions: In the term population considered healthy that developed transient neonatal hypoglycemia, an association was found with an Apgar score of 5 in the first minute, the first energy intake, TTN, polycythemia and skin-to-skin contact. The effects of variables that mostly influence metabolic glucose utilization can be seen in the long term in the motor skills and neurodevelopment of the population affected by this pathology. It is a wake-up call to be more vigilant of this group of newborns who, despite having a lower incidence of hypoglycemia, require surveillance and follow-up given the influence of various different factors.

Keywords: Hypoglycemia; Risk Factors; Newborn.

\section{Introducción}

La glucosa es el principal sustrato metabólico para asegurar el adecuado desarrollo fetal. Durante la vida intrauterina, todo el aporte de glucosa recibido por el feto proviene de la madre mediante un proceso de difusión facilitada a través de la placenta; asimismo, el feto ayuda a su homeostasis mediante la producción de insulina, lo que permite la euglucemia. Todo este equilibrio se altera después del nacimiento porque la fuente de aporte de glucosa disminuye y ejerce un mayor control la insulina fetal, lo que genera episodios de hipoglucemia (1). En neonatos sanos se han observado bajos niveles de glucemia durante las primeras 24 a 48 horas de vida posnatal; al igual que en la mayoría de los mamíferos, lo que permite concluir que puede representar un proceso de adaptación evolutivo a la vida extrauterina transitorio y no patológico $(1,2)$.

El cerebro depende casi exclusivamente de la glucosa para su adecuado funcionamiento y los recién nacidos sanos requieren tasas de infusión de glucosa más altas que los adultos $(2$ a 3 veces más por kilogramo de peso) con el fin de mantener la disponibilidad de glucosa debido a que, en la proporción corporal del neonato, el volumen ocupado por la masa cerebral es mayor que el resto de su cuerpo y, por ende, aumenta su consumo de glucosa (1). Cualquier situación que pueda comprometer el adecuado aporte de glucosa necesario para mantener la euglucemia llevará a hipoglucemia grave y persistente, que puede o no generar episodios convulsivos neonatales y desenlaces neurológicos desfavorables a largo plazo (2). Debido a lo anterior, se han realizado múltiples esfuerzos por detectar a los neonatos que se encuentran en riesgo de desarrollar hipoglucemia y compromiso neurológico para realizar un cribado durante su vida neonatal temprana, independientemente de la sintomatología.

El valor de referencia para considerar hipoglucemia varía ampliamente en la literatura (3), teniendo mayor vigencia los puntos de corte de la Asociación Americana de Pediatría (< 47 $\mathrm{mg} / \mathrm{dL}$ ) y la Asociación Española de Pediatría $(<50 \mathrm{mg} / \mathrm{dL})$. Sin embargo, no ha sido posible obtener un umbral exacto en el que se presente sintomatología neurológica, aunque en estudios se han identificado rangos que oscilan entre $<30 \mathrm{mg} / \mathrm{dL}$ hasta $40 \mathrm{mg} / \mathrm{dL}$ (4). La sintomatología se presenta comúnmente con letargia, hipotermia, taquicardia, taquipnea, alteración del estado de conciencia e incluso convulsiones, independientemente del valor de glucosa obtenido; sin embargo, suele ser inespecífica y puede asociarse con otras patologías presentes durante el período neonatal (5). Así mismo, se ha visto una disminución significativa en el perímetro cefálico a los 18 meses en los pacientes que presentaron episodios de convulsión por hipoglucemia $(6,7)$.

Reconocer a los neonatos con riesgo de hipoglucemia desde la edad prenatal es de vital importancia para disminuir las secuelas neurológicas y su estancia hospitalaria luego de una intervención temprana, la cual consiste en suplir una fuente adecuada y constante de glucosa y corregir su causa. Entre ellos se destacan el manejo con terapia antidiabética o antihipertensiva en las madres y patologías neonatales del paciente a término o pretérmino (restricción del crecimiento intrauterino, asfixia perinatal, sepsis, entre otros) (8-11).

El objetivo de nuestro estudio es determinar los factores de riesgo asociados con la hipoglucemia neonatal transitoria de recién nacidos a término. Esta población se considera sana según la literatura mundial, pero aun así tiene una incidencia del $2 \%-5 \%$ de desarrollar hipoglucemia; el reconocimiento de los factores de riesgo de esta población facilita su detección temprana e intervención, reduce las hospitalizaciones inne- 
cesarias por cuadros de hipoglucemia transitoria fisiológica, reconoce y anticipa tempranamente una hipoglucemia persistente y, en ambos casos, disminuye la probabilidad de progresar a secuelas neurológicas secundarias.

\section{Material y métodos}

Se trata de un estudio observacional analítico de tipo casos y controles en pacientes a término del Hospital Universitario Clínica San Rafael nacidos entre enero de 2015 y enero de 2019. Retrospectivamente, se recolectaron los datos de la historia clínica base Health On Line S.A (HeOn), para la definición de caso y control. Se definieron los casos como recién nacido vivo a término (entre 37 y 41 semanas) por la prueba de Ballard, con adecuado peso y talla para la edad gestacional y con diagnóstico de hipoglucemia neonatal (glucemia por tira glucométrica $<47 \mathrm{mg} / \mathrm{dL}$ ) y los controles como todo recién nacido vivo a término (entre 37 y 41 semanas) por la prueba de Ballard, con adecuado peso y talla para la edad gestacional sin diagnóstico de hipoglucemia neonatal transitoria.

Los criterios de inclusión fueron todos los recién nacidos a término vivos que ingresan a la unidad de cuidado básico, intermedio e intensivo neonatal del Hospital Universitario Clínica San Rafael con diagnóstico de hipoglucemia neonatal durante el período comprendido del estudio. Los criterios de exclusión fueron recién nacidos con diagnóstico prenatal de enfermedad metabólica o con variabilidad genética que afecten el metabolismo de la glucosa; episodios de hipoglucemia más allá de las primeras 48 horas de vida independientemente de su duración; todos los pacientes que permanecieron con valores de glucometría inferiores al punto de corte por más de 7 días, que constituyen pacientes con hipoglucemia prolongada; presencia de historias clínicas incompletas en las que no se documente su glucemia al ingreso ni durante las primeras 48 horas; hijos de madres con diagnóstico de diabetes gestacional; con embarazo de alto riesgo obstétrico según criterio del obstetra tratante.

Con el programa Real Statistics versión 6.9 de enero de 2020 se realizó el cálculo de la muestra y se tomó como referencia el artículo de Turner y colaboradores (12), con proporción de pacientes neonatos a término afectados por hipoglucemia neonatal transitoria 0,5 , con un Odds ratio (OR) de 1,5 (similar a lo descrito por Berttoloto y colaboradores en una población colombiana) (13), un intervalo de confianza (IC) del $95 \%$, potencia del $80 \%$ y proporción de casos hallados por dicho estudio de 0,3. Estos parámetros permitieron un cálculo muestral de un total de 35 pacientes para los casos y 35 controles, para lo que se realizó con un muestreo aleatorio simple. Las historias clínicas en físico y virtuales del sistema HeOn se exportaron al software R versión 3.6.2 de febrero de 2020 o la versión para Excel Real Statistics; se realizó prueba exacta de Fischer, su OR o chi cuadrado $\left(\chi^{2}\right)$ y su análisis factorial y correspondencia post hoc para determinar la asociación; en las variables continuas se realizó su descripción y se aplicaron las pruebas de Shapiro-Wilk y de la $U$ de Mann-Whitney para comparar casos y controles.

\section{Resultados}

La mayor proporción de pacientes en el estudio fue de sexo femenino (61,43\%), 20 casos y 23 controles y aunque el sexo no es un factor de riesgo, es 1,4 veces más probable que un neonato de sexo masculino presente hipoglucemia en nuestro estudio. A la vez, no se encontró asociación de la edad gestacional, peso, talla o perímetro cefálico (medidas antropométricas) con el desarrollo de hipoglucemia neonatal transitoria (Tabla 1).

Con respecto al Apgar a los 10 minutos, fue de 10 en la mayoría de los pacientes, en 32 casos (91,43 \%) y 33 controles $(94,29 \%)$. La asociación con hipoglucemia neonatal transito-

Tabla 1. Caracterización demográfica de los recién nacidos a término

\begin{tabular}{|c|c|c|c|c|c|}
\hline Variable & & $\mathbf{n}$ & Media & $\begin{array}{l}\text { Shapiro-Wilk } \\
\text { (valor } p \text { ) }\end{array}$ & $\begin{array}{l}\text { Prueba de la } U \text { de } \\
\text { Mann-Whitney/T }\end{array}$ \\
\hline $\begin{array}{l}\text { Edad gestacional (semanas } \\
\text { de gestación) }\end{array}$ & $\begin{array}{l}\text { Caso } \\
\text { Control }\end{array}$ & $\begin{array}{l}35 \\
35\end{array}$ & $\begin{array}{l}38,57 \\
38,63\end{array}$ & $\begin{array}{l}0,00245 \\
0,00069\end{array}$ & 0,7262 \\
\hline Talla $(\mathrm{cm})$ & $\begin{array}{l}\text { Caso } \\
\text { Control }\end{array}$ & $\begin{array}{l}35 \\
35\end{array}$ & $\begin{array}{l}50,69 \\
50,46\end{array}$ & $\begin{array}{l}0,18353 \\
0,02224\end{array}$ & 0,9952 \\
\hline Peso (g) & $\begin{array}{l}\text { Caso } \\
\text { Control }\end{array}$ & $\begin{array}{l}35 \\
35\end{array}$ & $\begin{array}{l}3162,94 \\
3054,57\end{array}$ & $\begin{array}{l}0,11559 \\
0,16597\end{array}$ & 0,5031 \\
\hline Perímetro cefálico $(\mathrm{cm})$ & $\begin{array}{l}\text { Caso } \\
\text { Control }\end{array}$ & $\begin{array}{l}35 \\
35\end{array}$ & $\begin{array}{l}35,11 \\
35,17\end{array}$ & $\begin{array}{l}0,00000 \\
0,00000\end{array}$ & 0,2045 \\
\hline
\end{tabular}


ria en los pacientes a término con un Apgar al minuto de 5 fue estadísticamente significativa en los casos sin configurar hipoxia neonatal en su desenlace. El promedio de la glucosa central fue de 40,8 mg/dL para los casos. La variable de policitemia, definida por un hematocrito $\geq 65 \%$ o hemoglobina $(\mathrm{Hb})$ $\geq 22 \mathrm{~g} / \mathrm{dL}$, se presentó en 10 pacientes de los casos (28,57 \%) y ninguno en los controles, y la taquipnea transitoria del recién nacido (TTRN) se presentó en $6(17,14 \%)$ de los casos y ninguno en los controles. La TTRN y la policitemia se asociaron con el desarrollo de hipoglucemia neonatal transitoria, variables a las cuales se les realizó una prueba exacta de Fisher en la que se mostró una asociación significativa (Tabla 2).

Las madres analizadas en el estudio tenían un promedio de edad de 24,31 años para los casos y de 26,86 años para los controles, y no mostró asociación alguna con el desarrollo de hipoglucemia neonatal transitoria. El número de gestación de las madres del estudio fue un promedio de 1 para los casos y 2 para los controles, pero no mostró asociación alguna con el desenlace de hipoglucemia neonatal transitoria. El índice de masa corporal (IMC) en promedio fue similar en los casos y los controles, en el rango de sobrepeso, sin mostrar asociación significativa para desarrollar hipoglucemia neonatal transitoria. No obstante, en nuestro estudio, es más probable que una madre con un IMC $>25 \mathrm{~kg} / \mathrm{m}^{2}$ tenga 1,2 veces más probabilidad de tener un hijo con hipoglucemia neonatal transitoria (Tabla 3).
Ninguna de las madres presentó código rojo; por tanto, no hay asociación alguna con el objeto de estudio. La hipertensión inducida por el embarazo y el tiempo de pinzamiento del cordón umbilical no estuvieron asociados con el desarrollo de hipoglucemia neonatal transitoria en nuestro estudio. Por otro lado, no se realizó apego a 7 (20\%) de los casos, mientras que la totalidad de los controles sí lo recibieron entre los 3 y 5 minutos, de acuerdo con nuestro protocolo institucional, y se pudo encontrar una asociación entre hipoglucemia neonatal transitoria y aquellos que no recibieron esta intervención (Tabla 3).

El primer aporte energético recibido por los pacientes con hipoglucemia neonatal transitoria fue en su mayoría por lactancia $(71,43 \%)$, de resto recibieron fórmula y líquidos dextrosados $(28,58 \%)$ al cursar con esta patología; en comparación con los controles que recibieron leche materna exclusiva al ser una institución amiga de la mujer y de la infancia (IAMI). Del total de los casos, 15 (42,86 \%) presentaron hipoglucemia sintomática y requirieron bolo de dextrosa al $10 \%$ a una dosis de $2 \mathrm{~mL} / \mathrm{kg}$ de peso, según el criterio médico (Tabla 4).

En los pacientes a término que presentaron hipoglucemia neonatal transitoria, la vía del parto fue la cesárea en 15 de los pacientes (42,8\%), mientras $20(57,14 \%)$ fueron vaginales. Aunque la vía del parto es un factor independiente de presentar hipoglucemia neonatal, en nuestro estudio pese a no encontrar asociación, se encontró que es 2,5 veces más probable

Tabla 2. Caracterización clínica y paraclínica de los recién nacidos a término

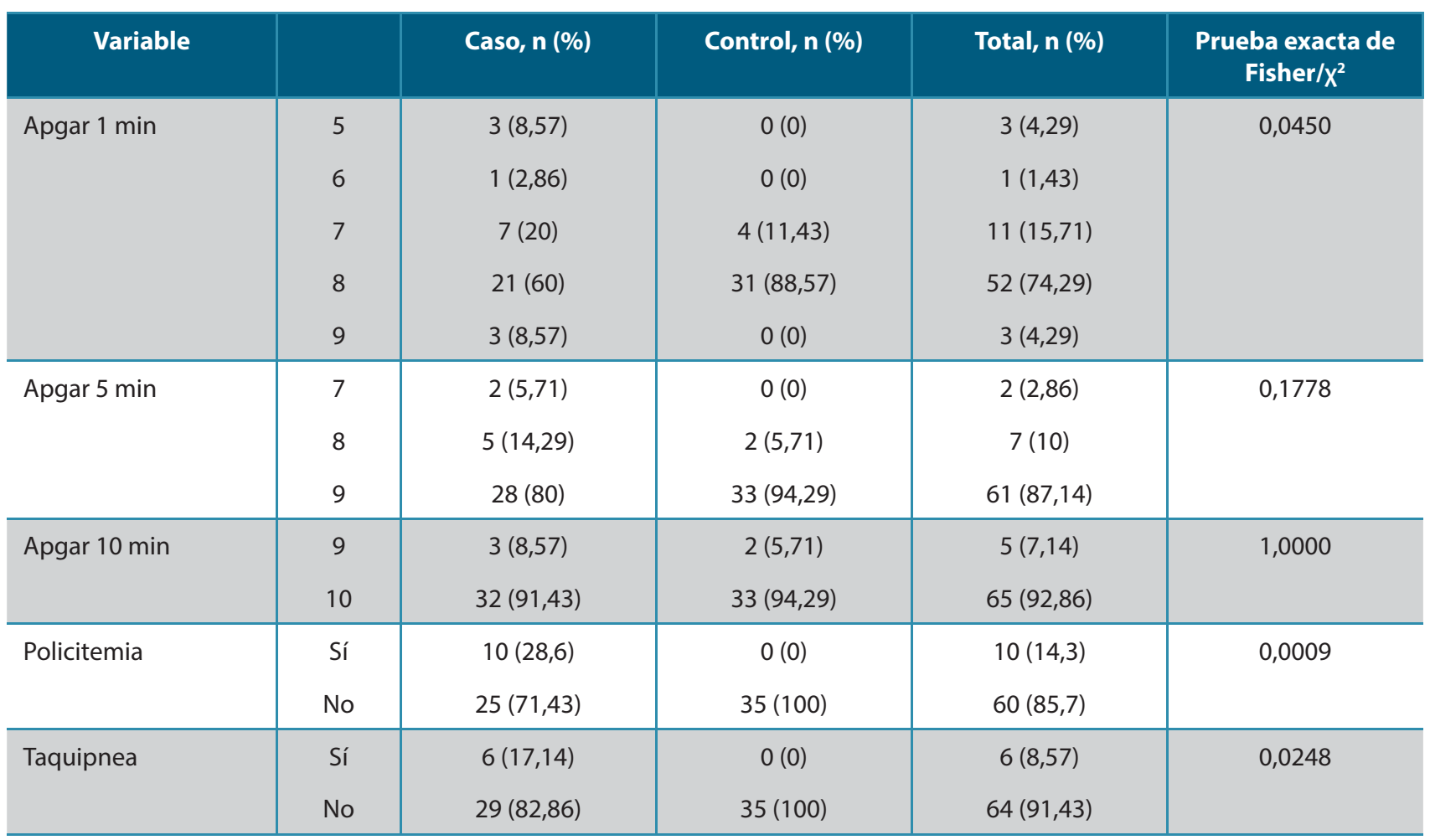


Tabla 3. Caracterización clínica de las madres

\begin{tabular}{|c|c|c|c|c|c|c|}
\hline Variable & & $\begin{array}{c}\text { Caso, n } \\
(\%)\end{array}$ & $\begin{array}{c}\text { Control, n } \\
(\%)\end{array}$ & $\begin{array}{c}\text { Total, n } \\
(\%)\end{array}$ & $\begin{array}{c}\text { Prueba exacta } \\
\text { de Fisher } / X^{2}\end{array}$ & OR \\
\hline Código rojo & No & $35(100)$ & $35(100)$ & $70(100)$ & $\mathrm{N} / \mathrm{A}$ & N/A \\
\hline IMC & $\begin{array}{l}\text { Normal } \\
\quad>25\end{array}$ & $\begin{array}{c}6(17,14) \\
29(82,86)\end{array}$ & $\begin{array}{c}5(14,29) \\
30(85,71)\end{array}$ & $\begin{array}{l}11(15,71) \\
59(84,29)\end{array}$ & 0,7425 & 1,24 \\
\hline HTA en el embarazo & $\begin{array}{l}\text { Sí } \\
\text { No }\end{array}$ & $\begin{array}{c}4(11,43) \\
31(88,57)\end{array}$ & $\begin{array}{c}1(2,86) \\
34(97,14)\end{array}$ & $\begin{array}{c}5(7,14) \\
65(92,86)\end{array}$ & 0,3565 & 4,3871 \\
\hline $\begin{array}{l}\text { Tiempo de pinzamiento del cordón: } \\
0 . \text { Precoz ( } 30-60 \mathrm{~s}) \\
\text { 1. Habitual ( } 1 \mathrm{~min}) \\
\text { 2. Tardío (2-3 min) }\end{array}$ & $\begin{array}{l}\text { Precoz } \\
\text { Habitual }\end{array}$ & $\begin{array}{c}3(8,57) \\
32(91,43)\end{array}$ & $\begin{array}{c}1(2,86) \\
34(97,14)\end{array}$ & $\begin{array}{c}4(5,71) \\
66(94,29)\end{array}$ & 0,6139 & 0,3137 \\
\hline Tiempo de apego & $\begin{array}{l}\text { No } \\
\text { Sí }\end{array}$ & $\begin{array}{c}7(20) \\
28(80)\end{array}$ & $\begin{array}{c}0(0) \\
35(100)\end{array}$ & $\begin{array}{l}7(10) \\
63(90)\end{array}$ & 0,0112 & $\mathrm{~N} / \mathrm{A}$ \\
\hline
\end{tabular}

HTA: hipertensión arterial; N/A: no aplica.

Tabla 4. Aporte energético del recién nacido a término

\begin{tabular}{|c|c|c|c|c|c|}
\hline Variable & & Caso, n (\%) & Control, n (\%) & Total, n (\%) & $\begin{array}{c}\text { Prueba exacta de } \\
\text { Fisher } / \mathrm{X}^{2}\end{array}$ \\
\hline Primer aporte energético & $\begin{array}{l}\text { Lactancia } \\
\text { Fórmula } \\
\text { Dextrosa }\end{array}$ & $\begin{array}{l}25(71,43) \\
5(14,29) \\
5(14,29)\end{array}$ & $\begin{array}{c}35(100) \\
0(0) \\
0(0)\end{array}$ & $\begin{array}{c}60(85,71) \\
5(7,14) \\
5(7,14)\end{array}$ & 0,0009 \\
\hline $\begin{array}{l}\text { Administración de bolo de } \\
\text { dextrosa al } 10 \%\end{array}$ & $\begin{array}{l}\text { Sí } \\
\text { No }\end{array}$ & $\begin{array}{l}15(42,86) \\
20(57,14)\end{array}$ & $\begin{array}{c}0(0) \\
35(100)\end{array}$ & $\begin{array}{l}15(21,43) \\
55(78,57)\end{array}$ & 0,0000 \\
\hline
\end{tabular}

Realizado por los investigadores, base HeOn.

que un niño con cesárea presente la patología en estudio. Ninguno de los casos tuvo parto instrumentado, por lo que no es posible establecer asociación alguna (Tabla 5).

\section{Discusión}

La hipoglucemia neonatal es una patología frecuente en el recién nacido con factores de riesgo y puede presentarse de forma transitoria o persistente; no obstante, su abordaje y diagnóstico en gran parte sigue siendo clínico. Muchos estudios han establecido que dichos factores pueden ser tanto maternos como neonatales, dentro de ellos se destacan el uso de antidiabéticos orales o antihipertensivos por parte de las madres y las patologías neonatales como restricción del crecimiento intrauterino (RCIU), asfixia perinatal y sepsis, entre otros. El retraso en el tratamiento de la hipoglucemia tiene consecuencias a largo plazo (aparición de convulsiones y retraso en el neurodesarrollo) que impactan en el pronóstico de estos pacientes (14).

En nuestro estudio se encontró que un Apgar a los 5 minutos en los recién nacidos a término muestra una asociación significativa con hipoglucemia; no obstante, como se mencionó en el estudio de cohorte de 2738 pacientes a término de Gilstrap y colaboradores, este puntaje por sí solo no define asfixia (patología asociada con el desarrollo de hipoglucemia) y no es satisfactorio para predecir el desenlace neurológico (15). Además, en el estudio de cohorte de 207 pacientes de Hosagasi y colaboradores no se encontró asociación alguna con el Apgar evaluado a los 5 minutos y su efecto en la inciden- 
Tabla 5. Vía de parto del recién nacido

\begin{tabular}{|l|l|c|c|c|c|c|}
\hline \multicolumn{1}{|c|}{ Variable } & Caso, n (\%) & Control, n (\%) & Total, n (\%) & $\begin{array}{c}\text { Prueba exacta de } \\
\text { Fisher/ } \mathbf{X}^{2}\end{array}$ \\
\hline Vía de parto & Cesárea & $15(42,86)$ & $8(22,86)$ & $23(32,86)$ & 0,1267 \\
& Vaginal & $20(57,14)$ & $27(77,14)$ & $47(67,14)$ & \\
\hline
\end{tabular}

Realizado por los investigadores, base $\mathrm{HeOn}$.

cia de hipoglucemia (16); lo que contrasta con lo encontrado en nuestros pacientes. Hay que tener en cuenta que la edad gestacional, medicamentos de uso materno, el tipo de anestésico administrado durante la cesárea y la persona que asignó el puntaje pueden influir en la puntuación. En nuestro estudio ningún paciente sufrió asfixia perinatal, algunos de los casos tuvieron adaptación conducida y como desenlace presentaron TTRN o policitemia, pero ninguno de los que presentaron Apgar al minuto 5 requirió ventilación mecánica invasiva.

Por otro lado, encontramos que el primer aporte energético recibido por el recién nacido tiene una asociación significativa con la hipoglucemia. En un estudio de 40 casos y 40 controles en la ciudad de Bogotá, Berttoloto y colaboradores encontraron un hallazgo similar, y muestran que el uso de fórmula láctea por ineficiente lactancia materna exclusiva sigue siendo el mayor factor de riesgo para la hipoglucemia neonatal transitoria (OR de 9) (13). En nuestro estudio no se pudo determinar el impacto de este factor dado que ningún control recibió fórmula y tampoco se realizó regresión logística incorporando las variables que aportaron el desenlace en el anterior. Sin embargo, en el estudio de Hosagasi y colaboradores (16) no se encontró dicha asociación con una muestra mayor, lo que evidencia una dicotomía en la literatura médica.

La disponibilidad de la glucosa se determina por la perfusión y su utilización individual, por lo que existen factores que impactan en su sensibilidad tales como sepsis neonatal, estrés perinatal, choque, enfermedad cardíaca congénita y enfermedad respiratoria (17). En nuestro estudio se mostró una asociación significativa de la TTRN con hipoglucemia, posiblemente relacionada con el aumento en la demanda metabólica, por exceso en la actividad de los músculos de la respiración y, por ende, el consumo o utilización de la glucosa. Se realizó una revisión exhaustiva de bases de datos para dicha asociación en pacientes a término sin factores de riesgo; no obstante, la mayoría de las publicaciones relacionaban el síndrome de dificultad respiratoria del recién nacido (en el cual la principal patología es la TTRN seguida de la enfermedad de membrana hialina) con pacientes en su mayoría prematuros tardíos y con hijos de madres diabéticas; en esta población la asociación de TTRN con hipoglucemia fue significativa, y además encontra- ron que requirieron más asistencia ventilatoria, ingreso en la unidad de cuidados intensivos neonatales y mayor estancia hospitalaria (18). Estos hallazgos son esperables, ya que globalmente la incidencia de complicaciones respiratorias en hijos de madres diabéticas es tan alta como $34 \%$, con una incidencia del síndrome de dificultad respiratoria del 4 \%-6 \% y un incremento de 2-3 veces más de riesgo respecto a hijos de madres no diabéticas $(19,20)$. El hallazgo obtenido en nuestro estudio da luz en cuanto a la elaboración de más estudios en población a término, la cual, a pesar de no tener factores de riesgo, mostró asociación entre TTRN e hipoglucemia.

Por su parte, pese a que la cesárea en nuestro estudio no mostró una asociación significativa con el desarrollo de hipoglucemia, como se planteaba en nuestra hipótesis de estudio (posiblemente por las limitaciones de los estudios retrospectivos), sí puede estar relacionado de forma indirecta con la hipoglucemia en forma de TTRN y, en consecuencia, con el consumo de la glucosa. Tal como describen Sweet y colaboradores, durante el parto por vía vaginal hay liberación de prostaglandinas que favorecen la distensión de los vasos linfáticos que remueven el líquido pulmonar del neonato (clearance) y aumenta la circulación pulmonar luego del primer respiro del recién nacido, pero debido a la cesárea este proceso fisiológico se pierde y persiste el líquido alveolar, lo que desencadena taquipnea y constituye un factor de riesgo para TTRN que, a su vez, se asocia con hipoglucemia (21). Nuestro estudio mostró que 15 casos (42,8\%) presentaron hipoglucemia cuando tuvieron parto por cesárea (OR 2,5), que es un dato no discriminable al compararlo con el estudio de Asa Ahimbisibwe y colaboradores (22), quienes incluían pacientes pretérmino tardíos y a término llevados a cesárea electiva, y cuyo objetivo era determinar la incidencia y gravedad de la morbilidad respiratoria en 1444 pacientes, de los cuales 54 presentaron morbilidad respiratoria y se encontró un riesgo aumentado (RR 2,14) para morbilidad respiratoria por cesárea electiva en $<38+$ 6 semanas (similar a la media de nuestra cohorte), respecto $a \geq 39$ semanas con una diferencia significativa. Lo anterior confirma los hallazgos en la literatura sobre TTRN y cesárea, y de forma indirecta explicar la presencia de hipoglucemia por consumo metabólico. 
De otro lado, la policitemia, en nuestro estudio, mostró una asociación significativa con el desarrollo de hipoglucemia en pacientes a término, lo que contrasta con la cohorte de 236 pacientes de Hopfeld-Fogel y colaboradores que incluía pacientes a término y cerca del término, de los cuales a 119 se les confirmó policitemia pero no establecieron asociación de la hipoglucemia con este hallazgo; no obstante, su cohorte está influenciada por 2 factores importantes: la inclusión de hijos de madres diabéticas y toxemia materna (que afecta la disponibilidad de la glucosa como metabolito). Sin embargo, sí establecieron que los casos de hipoglucemia neonatal en pacientes policitémicos se deben a factores fisiológicos (incluyeron pequeños para edad gestacional y grandes para edad gestacional), contrario a nuestra población que es a término y sin factores de riesgo; por lo que nuestro hallazgo es relevante debido a que se evaluó población sana (23). Adicionalmente, en la literatura se ha reportado una incidencia de policitemia de $2 \%-20 \%$ en recién nacidos a término, y es más frecuente en neonatos expuestos a hipoxia intrauterina, RCIU, pequeños para edad gestacional, hijos de madres con trastorno hipertensivo del embarazo (como algunas de nuestro estudio) e hijos de madres diabéticas o con trastornos tiroideos. Un estudio de relevancia histórica de Black y colaboradores encontró dicha asociación en una cohorte de 111 pacientes que incluyó un seguimiento hasta los 1-3 años y evidenciaron una mayor incidencia de anormalidades motoras y neurológicas en pacientes con policitemia e hipoglucemia (24), lo que apoya y ahonda en el estudio de la asociación de estas variables, como es el caso de nuestra muestra en población sana.

En cuanto a la variable materna que mostró asociación significativa, el tiempo de apego o contacto piel a piel se correlacionó con el desarrollo de hipoglucemia en aquellos que no la recibieron, esto también ha sido evaluado en un estudio cuasi experimental con 401 pacientes de Dalsgaard y colaboradores, donde a uno de los brazos que correspondía a recién nacidos a término, hijos de madres diabéticas manejadas únicamente con dieta, se les administró lactancia materna ininterrumpidamente con contacto piel a piel por 2 horas, encontrando que la proporción de eventos de hipoglucemia a las 2 horas era 2,5 veces y a las 4 horas 3,8 veces más altas en el grupo control que en el de intervención; en este estudio se administró fórmula láctea al grupo control y al de intervención, pero estos últimos recibieron menos sucedáneos de la leche y los que recibieron fue porque mantenían niveles bajos de glucosa a las 2 o 4 horas (25). En una población similar a la nuestra, Berttoloto y colaboradores encontraron que el contacto piel a piel es menor en pacientes producto de una cesárea y de forma aislada es un factor protector para hipoglucemia neonatal transitoria (OR 0,32) (13). Nuestros hallazgos son acordes a lo que reporta la literatura mundial, en la que el contacto piel a piel ha demostrado estabilizar la temperatura del recién nacido, el ahorro de glucosa y la disminución de la liberación de hormonas de estrés que aumentan la utilización de esta, procesos que se traducen en eventos de hipoglucemia (26). Este estudio incluyó una proporción similar de géneros en la muestra; sin embargo, está limitado por su tamaño y la variabilidad sujeta a una sola zona geográfica.

\section{Conclusiones}

Nuestro estudio aporta a la literatura actual la inclusión de población a término considerada sana que desarrolla hipoglucemia neonatal transitoria teniendo como variables asociadas el Apgar al minuto 5, el primer aporte energético recibido, la TTRN, la policitemia y el apego. Estas variables en su mayoría influyen en el metabolismo de utilización de la glucosa y, posteriormente como muestran otros estudios sobre el tema, sus efectos a largo plazo. Por lo que es un llamado a prestar atención a este grupo de recién nacidos que, aunque presentan hipoglucemia con menor incidencia, sí necesitan de nuestro acompañamiento y seguimiento. Se recomienda realizar estudios de tipo prospectivo con población a término con el fin de identificar factores que desencadenan la hipoglucemia e incluir otras variables que no se midieron en nuestro estudio porque no se podían obtener debido a la naturaleza retrospectiva y, de este modo, poder desarrollar un plan de acción dado el impacto posterior en el neurodesarrollo.

\section{Agradecimientos}

Agradecemos a todo el equipo de trabajo de la unidad de neonatología y ginecología del Hospital Universitario Clínica San Rafael de Bogotá, que contribuyen cada día a la mejora constante en la atención del recién nacido, y a nuestros docentes durante la formación académica, que han inculcado interés en los temas endocrinológicos. Así mismo, cabe mencionar la asesoría metodológica y estadística brindada por los docentes contratados por la Universidad Militar Nueva Granada: Dra. Ruth Vásquez Verano y Dr. Gerardo Ardila.

\section{Conflictos de interés}

Los autores en mención no tenemos conflicto de interés alguno, fue un trabajo en nuestro ejercicio académico. La fuente de financiación fue propia, haciendo uso de las instalaciones y elementos del Hospital Universitario Clínica San Rafael de Bogotá, bajo el apoyo de la Universidad Militar Nueva Granada. 


\section{Referencias}

1. Adamkin DH. Neonatal hypoglycemia. Semin Fetal Neonatal Med 2017;22(1):36-41.

2. Hiersch L, Krispin E, Aviram A, Wiznitzer A, Yogev Y, Ashwal E. Effect of Meconium-Stained Amniotic Fluid on Perinatal Complications in Low-Risk Pregnancies at Term. Am J Perinatol. 2016:33(4):378-84.

3. Committee on Fetus and Newborn, Adamkin DH. Postnatal glucose homeostasis in late-preterm and term infants. Pediatrics. 2011;127(3):575-9.

4. Duvanel CB, Fawer CL, Cotting J, Hohlfeld P, Matthieu JM. Long-term effects of neonatal hypoglycemia on brain growth and psychomotor development in small-for-gestational-age preterm infants. J Pediatr. 1999;134(4):492-8.

5. McKinlay CJD, Alsweiler JM, Anstice NS, Burakevych N, Chakraborty A Chase JG, et al. Association of Neonatal Glycemia With Neurodevelopmental Outcomes at 4.5 Years. JAMA Pediatr. 2017;171(10):972-983.

6. Thompson-Branch A, Havranek T. Neonatal Hypoglycemia. Pediatr Rev. 2017;38(4):147-157.

7. Stomnaroska O, Petkovska E, Jancevska S, Danilovski D. Neonatal Hypoglycemia: Risk Factors and Outcomes. Pril (Makedon Akad Nauk Umet Odd Med Nauki). 2017;38(1):97-101.

8. Flores-le Roux JA, Sagarra E, Benaiges D, Hernandez-Rivas E, Chillaron JJ, Puig de Dou J, et al. A prospective evaluation of neonatal hypoglycaemia in infants of women with gestational diabetes mellitus. Diabetes Res Clin Pract. 2012;97(2):217-22.

9. McKinlay CJD, Chase JG, Dickson J, Harris DL, Alsweiler JM, Harding JE. Continuous glucose monitoring in neonates: a review. Matern Health Neonatol Perinatol. 2017;3:18.

10. Cornblath M, Odell GB, Levin EY. Symptomatic neonatal hypoglycemia associated with toxemia of pregnancy. J Pediatr. 1959;55:545-62.

11. Coors SM, Cousin JJ, Hagan JL, Kaiser JR. Prophylactic Dextrose Gel Does Not Prevent Neonatal Hypoglycemia: A Quasi-Experimental Pilot Study. J Pediatr. 2018;198:156-161.

12. Turner D, Monthé-Drèze C, Cherkerzian S, Gregory K, Sen S. Maternal obesity and cesarean section delivery: additional risk factors for neonatal hypoglycemia? J Perinatol. 2019;39(8):1057-1064.

13. Berttoloto-Cepeda A, Vargas-Vaca Y, Guzmán-Cruz P, Murillo-Casas A, Muñoz-Peña L. Factores asociados a hipoglucemia neonatal transitoria en recién nacidos sanos, en el Hospital Universitario San Ignacio, estudio de casos y controles. Universitas Médica. 2017;58(3):1-5.

14. García-Patterson A, Aulinas A, María MÁ, Ubeda J, Orellana I, Ginovart G, et al. Maternal body mass index is a predictor of neonatal hypoglycemia in gestational diabetes mellitus. J Clin Endocrinol Metab. 2012;97(5):1623-8.
15. Gilstrap LC 3rd, Leveno KJ, Burris J, Williams ML, Little BB. Diagnosis of birth asphyxia on the basis of fetal $\mathrm{pH}$, Apgar score, and newborn cerebral dysfunction. Am J Obstet Gynecol. 1989;161(3):825-30.

16. Hosagasi NH, Aydin M, Zenciroglu A, Ustun N, Beken S. Incidence of hypoglycemia in newborns at risk and an audit of the 2011 American academy of pediatrics guideline for hypoglycemia. Pediatr Neonatol. 2018;59(4):368-374.

17. Puchalski ML, Russell TL, Karlsen KA. Neonatal Hypoglycemia: Is There Sweet Spot? Crit Care Nurs Clin North Am. 2018;30(4):467-480.

18. Fung GP, Chan LM, Ho YC, To WK, Chan HB, Lao TT. Does gestational diabetes mellitus affect respiratory outcome in late-preterm infants? Early Hum Dev. 2014;90(9):527-30.

19. Michael Weindling A. Offspring of diabetic pregnancy: short-term outcomes. Semin Fetal Neonatal Med. 2009;14(2):111-8.

20. Cordero L, Treuer SH, Landon MB, Gabbe SG. Management of infants of diabetic mothers. Arch Pediatr Adolesc Med. 1998;152(3):249-54.

21. Sweet LR, Keech C, Klein NP, Marshall HS, Tagbo BN, Quine D, et al. Respiratory distress in the neonate: Case definition \& guidelines for data collection, analysis, and presentation of maternal immunization safety data. Vaccine. 2017;35(48 Pt A):6506-6517

22. Ahimbisibwe A, Coughlin K, Eastabrook G. Respiratory Morbidity in Late Preterm and Term Babies Born by Elective Caesarean Section. J Obstet Gynaecol Can. 2019;41(8):1144-1149.

23. Hopfeld-Fogel A, Kasirer Y, Mimouni FB, Hammerman C, Bin-Nun A. Neo natal Polycythemia and Hypoglycemia in Newborns: Are They Related? Am J Perinatol. 2020. Publicación electrónica antes de la impresión.

24. Black VD, Lubchenco LO, Luckey DW, Koops BL, McGuinness GA, Powell DP et al. Developmental and neurologic sequelae of neonatal hyperviscosity syndrome. Pediatrics. 1982;69(4):426-31.

25. Dalsgaard BT, Rodrigo-Domingo M, Kronborg H, Haslund H. Breastfeeding and skin-to-skin contact as non-pharmacological prevention of neonatal hypoglycemia in infants born to women with gestational diabetes; a Danish quasi-experimental study. Sex Reprod Healthc. 2019;19:1-8.

26. Moore ER, Bergman N, Anderson GC, Medley N. Early skin-to-skin contact for mothers and their healthy newborn infants. Cochrane Database Syst Rev. 2016;11(11):CD003519. 


\section{SANOFI У}

\section{AYUDE A SUS PACIENTES A LOGRAR LA META DE HbA1c}

$\mathbf{7 4 \%}$ de los pacientes que venían descontrolados con ADOs, alcanzaron la meta de $\mathrm{HbAlc}$ con una sola inyección al día de SOLIQUA ${ }^{\circledR 1}$.

SOLIQUA ${ }^{\circledR}$ reduce en promedio $-2,9 \%$ * de $\mathrm{HbAlc}$ en el sub-grupo de pacientes con $\mathrm{HbAlc}>9 \%$ al inicio del tratamiento?

*Promedio de reducción en pacientes tratados previamente con ADOs con HbAlc > 9\%; el promedio de reducción de HbAlc del total de pacientes descontrolados tratados previamente con ADOs fue - 1,6\%.

Conozca más sobre SOLIQUA ${ }^{\boxplus}$ descargando SANOFIMED+

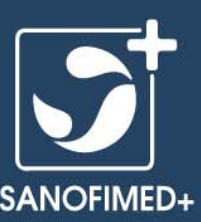

Google play

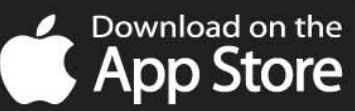

\section{SOLIOUA \\ insulina glargina $(100 \mathrm{U} / \mathrm{mL})$ \& lixisenatida}

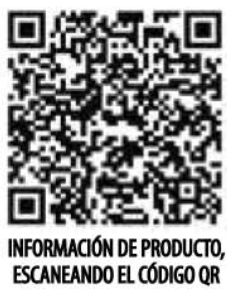

